\title{
Tsafon
}

Revue d'études juives du Nord

$81 \mid 2021$

Des synagogues à travers les âges Lieux de prières, lieux d'études et autres fonctions

\section{La question des Affranchis dans les synagogues de la période du second Temple}

Yaël Escojido

\section{(2) OpenEdition}

Journals

Édition électronique

URL : https://journals.openedition.org/tsafon/3629

DOI : $10.4000 /$ tsafon.3629

ISSN : 2609-6420

Éditeur

Association Jean-Marie Delmaire

Édition imprimée

Date de publication : 1 juillet 2021

Pagination : 23-38

ISSN : $1149-6630$

Référence électronique

Yaël Escojido, «La question des Affranchis dans les synagogues de la période du second Temple », Tsafon [En ligne], 81 | 2021, mis en ligne le 01 juillet 2021, consulté le 15 septembre 2021. URL : http:// journals.openedition.org/tsafon/3629; DOI : https://doi.org/10.4000/tsafon.3629 


\title{
La question des Affranchis dans les synagogues de la période du second Temple
}

\author{
Yaël Escojido*
}

Les sources qui relatent de la fin du second Temple, les écrits de Flavius Josèphe et du Nouveau Testament, révèlent l'existence de nombreuses synagogues en Terre sainte. De plus, bien que le Temple soit, au début de l'ère chrétienne, le lieu de culte central, il existait aussi dans la ville de Jérusalem quelques synagogues ${ }^{1}$. L'une d'entre elles nommée

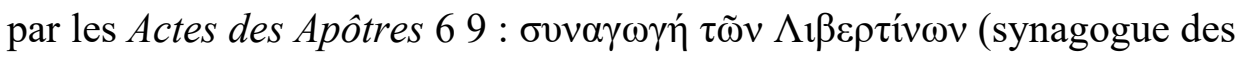
Affranchis) suggère un certain lien avec d'anciens esclaves.

Nous proposons, dans cette étude, d'examiner les raisons pour lesquelles des affranchis de l'esclavage avaient construit près du Temple de Jérusalem leur propre synagogue. Se pouvait-il qu'un Juif, auquel les aléas de la vie avaient imposé l'assujettissement, fût dans l'impossibilité, une fois libéré, de fréquenter une synagogue de Juifs qui, pour leur part, étaient toujours restés libres? Cette interrogation pourrait expliquer pourquoi d'anciens esclaves juifs se regroupaient dans une synagogue distincte des autres synagogues de Jérusalem. Ou bien, pourrions-nous envisager les choses sous un angle différent en suggérant que la synagogue des Affranchis était fréquentée par d'anciens esclaves païens, lesquels avaient embrassé la religion de leurs maîtres juifs et, ainsi, avaient fondé leur propre synagogue.

Pour répondre à ces questions nous procéderons par étapes. Nous commencerons par un examen des rôles et fonctions de la synagogue à la

\footnotetext{
* The Israel \& Golda Koschitzky Department of Jewish History and Contemporary Jewry, Bar Ilan University. Cet article est une version adaptée d'un chapitre de ma thèse de doctorat, intitulée : Jewish Slavery in the Second Temple Period in the Light of Greek Sources, Université Bar-Ilan, Ramat-Gan, Israël.

${ }^{1} T B$, Ketubot 105a fait savoir que 394 synagogues se trouvaient à Jérusalem à l'époque du second Temple. TJ, Méguila III 1, 73d mentionne 480 synagogues à Jérusalem avant la destruction du Temple par les Romains.
} 
fin du second Temple à travers les sources littéraires et archéologiques de cette période ; et plus principalement, sa fonction, peut-être un peu moins connue, dans l'affranchissement d'esclaves païens dont les maîtres étaient juifs. Puis, nous continuerons par une brève présentation d'un groupe différent de synagogues, à Jérusalem et à Rome, celles qui relèvent d'anciens esclaves. Enfin, on tentera de définir les origines de la synagogue des Affranchis à Jérusalem, ainsi que les circonstances historiques qui amenèrent à son établissement.

\section{Rôles et fonctions de la synagogue à la fin du second Temple}

Avant d'entamer le débat sur les rôles et fonctions de la synagogue, il convient d'abord de comprendre les termes grecs employés par le monde synagogal juif au $\mathrm{I}^{\mathrm{er}}$ siècle ap. J-C, apparaissant dans les sources littéraires et épigraphiques que nous utiliserons par la suite.

Nous avons choisi de nous en tenir aux conclusions de Rachel

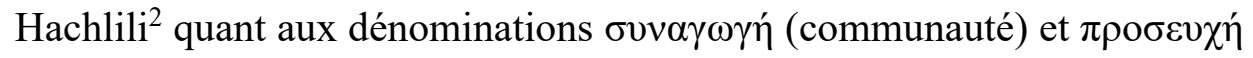
(édifice de prières) et la définition d'une synagogue au $\mathrm{I}^{\mathrm{er}}$ siècle ap. J-C :

1) L'utilisation des deux termes est attestée avant et après la destruction du Temple de Jérusalem.

2) L'apparition d'un terme ou d'un autre ne révèle aucune distinction, en matière de chronologie, ni de distinction de structure d'édifice voire de différentiation d'utilisation en Palestine ou en diaspora.

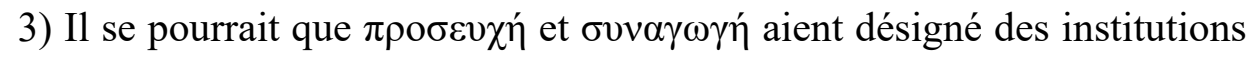
différentes et cela, à la même période.

4) Ces deux termes ont connu un développement certain quant à leur signification religieuse depuis le III ${ }^{\text {ème }}$ siècle avant notre ère, lorsqu'ils apparaissent pour la première fois et ceci jusqu' à la période byzantine.

Nous proposons maintenant de présenter certaines fonctions de la synagogue à l'époque romaine et, plus en détail, celle qui lui était attribuée lors d'affranchissement d'esclaves par des maîtres juifs.

Lee Levine affirme qu'à la fin de la période du second Temple, c'est dans la synagogue que se concentrait la vie juive ${ }^{3}$. C'est là qu'on y rendait les sentences pénales et la justice en général. La synagogue était aussi le centre de l'organisation financière de la communauté ainsi que son centre

\footnotetext{
${ }^{2}$ Rachel Hachlili, «The Origin of the Synagogue : A Re-Assessment », Journal for the Study of Judaism in the Persian, Hellenistic, and Roman Period, 28, 1997, p. 40.

${ }^{3}$ Lee L. Levine, "The Form and Content of the Synagogue in the Second Temple Period », dans A. Kasher et al. (eds.), Synagogues in Antiquity, Jerusalem, Yad Izhak ben Zvi, 1987, p.18. (hébreu).
} 
politique. Mais c'était surtout le lieu où l'on enseignait les lois juives et où on les interprétait. Les témoignages de Philon d'Alexandrie, de Flavius Josèphe et ceux du Nouveau Testament coïncident pour rapporter que telle fut la fonction principale de la synagogue ${ }^{4}$.

Philon, qui témoigne de la vie juive à Alexandrie pendant la période romaine, indique que les synagogues étaient des écoles dans lesquelles étaient enseignées, le jour du shabbath, la vertu et la philosophie ancestrale $^{5}$. Ce qui permet à Heather Mc Kay d'affirmer que l'interprétation des textes de la Loi n'était pas seulement à but religieux et que dans les synagogues étaient évoqués des sujets d'ordre moral, social et autres ${ }^{6}$.

Examinons maintenant les sources épigraphiques, trouvées dans l'ancien royaume du Bosphore, qui renseignent sur la fonction des synagogues lors de la manumission d'esclaves par des maîtres juifs. Ainsi les inscriptions juives des cités hellénistiques de Panticapée, Phanagorie et Gorgippia $^{7}$, relèvent d'actes d'affranchissement, gravés sur du marbre, datés entre le $\mathrm{I}^{\mathrm{er}}$ et le $\mathrm{II}^{\mathrm{e}}$ siècles ap. J-C et caractérisés par trois points distincts :

1) L'affranchissement a lieu dans une synagogue (de type : proseuque).

2) La communauté juive ${ }^{9}$ supervise l'acte d'affranchissement.

3) L'esclave affranchi a pour obligation de fréquenter régulièrement la maison de prière (de type : proseuque) de son ancien maître ${ }^{10}$.

\footnotetext{
${ }^{4}$ Les sources suivantes font mention de lecture, enseignement, ou simplement de la présence de livres de la Tora dans une synagogue. Cf. Philon d'Alexandrie, Somn. II 127, Opif. 128, Hypoth. VII 12-13, Legat. 156-157, Mos. II 215-216, Spec. Leg. II 62, Contempl. 30-31, Prob. 80-83 ; Josephus, Bellum Judaicum II 290-292. Mc I 21, 39, VI 2 ; Mt IV 23, IX 35, XIII 54 ; Lc IV 15, 16-28, 31-32, 44, VI 6, XIII 10, Je VI 60, XVIII $20 ;$ Act. Ap. IX 20, XIII 5, 14-15, XIV 1, XV 21, XVII 2-3, 10-11, 17, XVIII 4, 26, XIX 8. Ajoutons à ces sources littéraires, l'inscription de Théodote qui présente la construction d'une $\sigma v v \alpha \gamma \omega \gamma$ ń pour la « lecture de la Loi et l'enseignement des préceptes », dans JeanBaptiste Frey (ed.), Corpus Inscriptionum Judaicarum, I-II, Rome, Pontificio Instituto di Archeologia Cristiana, 1936, 1952 (= CIJ), Ibid, II, 1404, p. 332-335.

${ }^{5}$ Vita Moses II 216.

${ }^{6}$ Heather Mc Kay, Sabbath and Synagogue: The Question of Sabbath Worship in Ancient Judaism, Leiden, Brill, 1994, p. 66. Cf. aussi Lee L.Levine, The Ancient Synagogue, New Haven-London, Yale University Press, 2005 ( $2^{\mathrm{e}}$ édition), p. 155-156. Levine admet cette même conclusion pour les synagogues de Judée et de la diaspora.

${ }^{7}$ CIRB (Corpus inscriptionum regni Bosporani) 70, 71, 72, 73, 985, 1123, 1127, SEG 43.

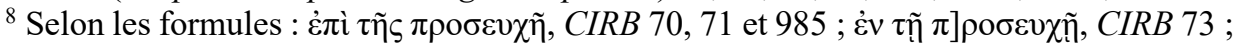

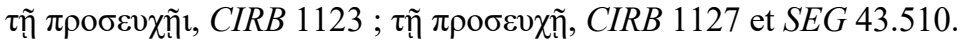

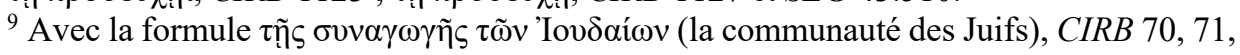
72,74 et $S E G 43.510$.

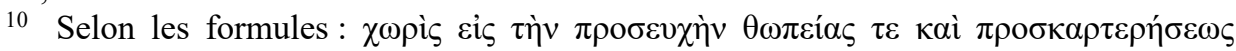
(excepté [son devoir] envers la synagogue [qu'il devra servir] avec dévouement et
} 
Il semble donc que dans la diaspora gréco-romaine, les Juifs affranchissaient leurs esclaves étrangers dans une synagogue, à l'instar de leurs voisins païens qui accomplissaient l'affranchissement dans un temple païen. Pour autant, si les actes d'affranchissement dans le temple d'Apollon, ainsi que le montrent les nombreux exemples delphiens, constituaient une vente fictive par laquelle le propriétaire libérait un ou plusieurs esclaves en le vendant au dieu contre une somme d'argent déposée au temple par l'esclave lui-même, ceux retrouvés dans les synagogues de l'ancien royaume du Bosphore ne mentionnent aucun paiement ou vente fictive, en contrepartie de l'affranchissement. Cependant, tant pour les affranchissements synagogaux, que pour ceux gravés dans les temples à Delphes, on publiait un contrat qui différait la liberté de l'esclave. Il s'agissait d'affranchissements avec clause de paramona $^{11}$, astreignant $1^{\prime}$ 'esclave affranchi à demeurer auprès et au service du maître pour une période déterminée. Cette clause de contrat se manifestait notamment dans les actes d'affranchissement juifs, contraignant l'esclave païen affranchi à fréquenter la synagogue de son maître.

En quoi consistait l'obligation de l'affranchi envers la synagogue de son maitre ?

Dans les actes d'affranchissement du royaume du Bosphore cette obligation est soulignée par deux termes grecs que Joseph Derenbourg traduit par: «assiduité et dévouement» ${ }^{12}$. Cependant, aucune information, ne nous est parvenue quant aux gestes, services, cérémonie ou liturgie que ces esclaves libérés devaient pratiquer afin de prouver leurs dévouement et assiduité à la synagogue. Il est pourtant clair que ces deux notions devaient se manifester lors de la fréquentation des esclaves affranchis dans le lieu de prière de leurs maîtres, conformément à la formule d'affranchissement : « il sera loisible [aux affranchis] d'aller en quelque lieu $[\ldots]$ sans empêchement et sans entrave [...] excepté pour ce qui regarde la synagogue à laquelle ils devront dévouement et assiduité

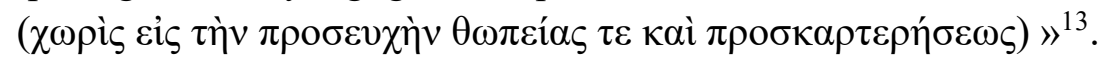

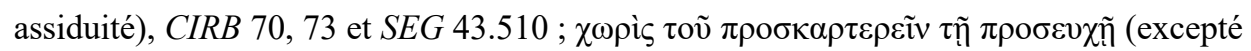
son dévouement à la synagogue), $C I R B 71$

${ }^{11} \Pi \alpha \rho \alpha \mu o v \eta ́$ : obligation de continuer à servir son maître, pour un esclave dont l'affranchissement proclamé est néanmoins différé.

12 Joseph Derenbourg, «Les inscriptions grecques juives au nord de la mer Noire »,

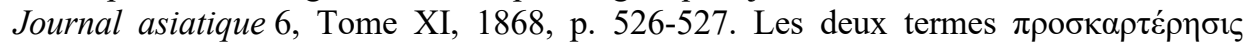
(assiduité) et $\theta \omega \pi \varepsilon i ́ \alpha$ (dévouement) se trouvent associées dans les inscriptions CIRB 70,

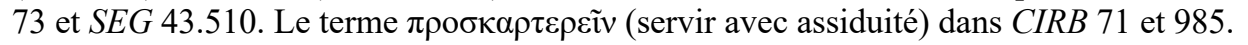
${ }^{13}$ Ibid. 
Le cas d'affranchissement dans une synagogue impliquait-il donc l'engagement de l'esclave païen dans la religion de son maître juif?

La formulation $\chi \omega \rho i \varsigma ~ \tau о \tilde{~} \pi \rho о \sigma \kappa \alpha \rho \tau \varepsilon \rho \varepsilon \tilde{v} \tau \tilde{n} \pi \rho о \sigma \varepsilon v \chi \tilde{n}$ rappelle un passage qui lui est presque contemporain, évoqué par saint Paul dans son Épître aux Romains «Avec la joie de l'espérance, constants dans la tribulation, assidus à la prière ${ }^{14}$. Il semble donc que l'expression $\pi \rho \circ \sigma \kappa \alpha \rho \tau \varepsilon \rho \varepsilon \bar{v} \tau \tilde{n} \pi \rho \circ \sigma \varepsilon v \chi \tilde{n}$ corresponde tant à l'assiduité requise dans la maison de prière qu'à la prière elle-même.

Pour la majorité des chercheurs, il est donc évident que l'obligation des esclaves libérés envers la synagogue de leur maître relevait d'un acte religieux ainsi que d'une participation régulière au culte juif synagogal. Pour J. Derenbourg et D. Dan'shin, cette conclusion indique en outre que la cérémonie d'affranchissement dans la synagogue correspondait en fait à la conversion de l'esclave païen au judaïsme ${ }^{15}$.

Le but de cet article ne visant pas à traiter de manière détaillée la riche documentation épigraphique juive du Bosphore mais plutôt d'exposer la fonction de la synagogue lors de cérémonie d'affranchissement, nous retiendrons les données suivantes :

1) Si les écrits de la Bible ne précisent aucune loi concernant la manumission d'esclaves étrangers ${ }^{16}$, il semble que les maîtres juifs en diaspora adoptèrent, à cet effet, les coutumes grecques des pays dans lesquels ils vivaient. La synagogue, sous sa forme de proseuque, se substitue ainsi aux temples dans ces «formes païennes judaïsées » ${ }^{17}$ d'affranchissement d'esclave.

2) Si les actes d'affranchissement que nous avons évoqués sont à caractère séculier comme l'a souligné Rachel Zelnick-Abramovitz ${ }^{18}$, la cérémonie d'affranchissement dans une synagogue leur attribue une forme sacrée. Ceci permet de signaler l'aspect sacré de la synagogue aux yeux des Juifs de la communauté de l'ancien royaume du Bosphore.

\footnotetext{
${ }^{14}$ Rom. XII 12.

${ }^{15}$ J. Derenbourg, «Les inscriptions grecques juives », op. cit., p. 536 ; D. I. Dan'shin, "The Jewish Community of Phanagoria », Ancient Civilizations from Scythia to Siberia 3, 1997, p. 140.

${ }^{16}$ Selon la Bible un esclave païen demeurait à jamais la propriété de son maître : לעל

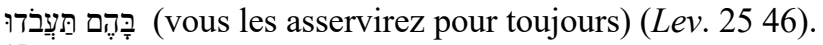

${ }^{17}$ Jean Juster, Les Juifs dans l'empire romain, leur condition juridique, économique et sociale, II, Paris, P. Geuthner, 1914, p. 82.

${ }^{18}$ Rachel Zelnick-Abramovitz, Not Wholly Free, The Concept of Manumission and the Status of Manumitted Slaves in the Ancient Greek World, Leiden-Boston, Brill, 2005, p. 89 , n. 56.
} 
3) La fréquentation régulière de la synagogue par des affranchis nous informe sur le degré d'absorption des us et coutumes juives par les esclaves païens.

Avant de traiter en détail, la question des affranchis dans la synagogue de Jérusalem telle qu'elle est mentionnée dans Act. Ap. VI 9, il paraît utile de donner un aperçu général d'autres synagogues de l'époque romaine qui mentionnent esclaves ou affranchis. D'abord la synagogue des Vernaculi à Rome puis celle de Jérusalem signalée dans l'inscription de Théodote fils de Ouettènos. Le but étant d'abord de définir l'origine servile des membres ou fondateurs de ces deux synagogues puis, si les sources dont nous disposons nous le permettent, d'examiner en quoi consistait leur organisation.

\section{Synagogues, esclaves et affranchis}

$\mathrm{Au}$ début de l'ère chrétienne, la population juive de Rome se répartissait en de nombreuses synagogues. Le terme synagogue désigne alors toujours la communauté juive et non l'édifice cultuel ${ }^{19}$. Du reste, jusqu'à présent aucune ruine de synagogue n'a émergé des vestiges archéologiques de la Rome antique.

Dans son article sur les communautés juives de Rome, Jean-Baptiste Frey regroupe donc les inscriptions épigraphiques relatant de synagogues / communautés en 3 groupes distincts d'après leur dénomination ${ }^{20}$ :

1) Les synagogues dénommées d'après les personnages célèbres de Rome, comme la synagogue des Augustenses, mentionnée dans le grand éloge que fit Philon d'Alexandrie de l'empereur Auguste et des faveurs qu'il accorda aux Juifs de Rome ${ }^{21}$. Ou encore la synagogue des Aggripenses d'après Marcus Vipsanius Aggrippa (62-12 av. J-C) qui manifesta à l'égard des Juifs des sentiments identiques à ceux de l'empereur Auguste ${ }^{22}$. Ces communautés étaient formées selon le système du patronatus, connu également par d'autres inscriptions païennes, qui consistait à placer une corporation ou des collegia de toute sorte sous le patronage d'un personnage influent, lequel accordait en retour sa protection.

\footnotetext{
19 Jean-Baptiste Frey, «Les communautés juives à Rome », Recherches de science religieuse, $\mathrm{n}^{\circ} 20,1930$, p. 282-283, n. 49.

${ }^{20}$ CIJ I, op. cit., p. LXX-LXXXI. Les travaux de Frey portent sur 13 synagogues dans la Rome antique.

${ }^{21}$ Legatio ad Caium 23 155-158.

${ }^{22}$ Flavius Josèphe, Antiquités Juives XVI 14.
} 
2) Les synagogues dénommées d'après les quartiers de Rome du fait de leur emplacement; Frey en dénombre trois ${ }^{23}$.

3) Les synagogues dénommées d'après l'origine de leurs membres fondateurs. Outre la synagogue de Tripolis (Lybie), les inscriptions romaines renseignent sur la synagogue des Hébreux et celle des Vernaculi, signalée par quatre sources épigraphiques ${ }^{24}$. Il semble que la qualification explicite d'une communauté d'Hébreux désigne «la synagogue des Hébreux » comme le groupement juif plus ancien de Rome ${ }^{25}$.

Pour la synagogue des Vernaculi, comme le suggère son nom dérivé du latin vernal (esclave), l'origine servile de cette communauté a été envisagée. Selon Théodore Reinach, les premiers membres de cette communauté devaient être nés dans la servitude puis ensuite affranchis ${ }^{26}$. Selon Franz Cumont, il s'agissait probablement d'esclaves de la maison impériale ${ }^{27}$. Pieter van der Host souligne que le vernaculus pourrait

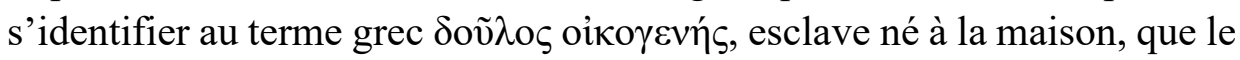
monde gréco-romain différenciait du prisonnier de guerre ou de l'esclave acheté au marché2 ${ }^{2}$. E. Mary Smallwood suggère que la synagogue des Vernaculi reflétait le témoignage de Philon d'Alexandrie selon lequel la grande majorité de la communauté juive de Rome était constituée d'anciens esclaves et d'affranchis ${ }^{29}$. Cependant, comme le suggère $P$. van der Horst, il semble peu probable que les Juifs de Rome aient voulu

\footnotetext{
${ }^{23}$ CIJ I., p. LXXIII-LXXV. Ce sont: la synagogue des Siburenses, de la zone urbaine romaine Suburra, quartier populaire où les Juifs étaient installés ; celle des Campeses, au quartier du Champs de Mars; et celle des Calcarenses, plus difficile à localiser.

${ }^{24}$ La synagogue des Vernaculi est indiquée dans les sources épigraphiques sous les

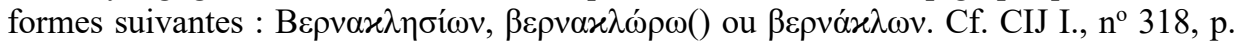
$249-250 ; n^{\circ} 383$, p. 297-298; $n^{\circ} 398$, p. 307-308; $n^{\circ} 494$, p. 359-360. Les trois premières inscriptions concernant la synagogue des Vernaculi ont été trouvées dans la grande catacombe de Monteverde à Rome, lieu de sépulture des quartiers de la rive droite du Tibre. L'inscription, CIJ, I 494 provient quant à elle du quartier du Transtevere.

${ }^{25}$ Romano Penna, «Les Juifs à Rome au temps de l'apôtre Paul », New Testament Studies, 28, 1982, p. 328 ; Harry J. Leon, The Jews of Ancient Rome, Philadelphia, Jewish Publication Society of America, 1960, p. 147-149; Pieter W. van der Horst, Ancient Jewish Epitaphs : An introductory survey of a millennium of jewish funerary epigraphy (300 BCE-700 CE), Kampen, Pharos, 1991, p. 87.

${ }^{26}$ Théodore Reinach, «Le cimetière juif de Monteverde », Revue des études juives, 71, 1920, p. 121.

${ }^{27}$ Franz Cumont, « Catacombes juives de Rome », Syria, Archéologie, Art et histoire, 2, 1921, p. 146.

${ }^{28}$ Pieter W. van der Horst, Ancient Jewish Epitaphs, op.cit., p. 88.

${ }^{29}$ E. Mary Smallwood, The Jews under Roman Rule : from Pompey to Diocletian, Leiden, Brill, 1981, p. 234-235, sur la remarque de Philon, Legatio 155 : P $\pi \lambda \varepsilon i ́ o v \varsigma \alpha a ̉ \pi \varepsilon \lambda \varepsilon v \theta \varepsilon \rho \omega \theta \varepsilon \dot{v} v \tau \varepsilon \varsigma$ (La plupart [des Juifs] romains étaient des affranchis).
} 
rappeler leurs origines serviles au travers du nom de leur synagogue ${ }^{30}$. C'est pourquoi, à l'hypothèse d'une communauté constituée d'esclaves affranchis, nous préférons les conclusions de Harry Leon indiquant que le mot vernaculus signifie « natif » ou « indigène », par opposition au terme d'immigrant récent et qui n'est aucunement lié au monde de l'esclavage ${ }^{31}$.

Par conséquent, la synagogue des Vernaculi se distinguait non par le statut social de ses membres mais davantage par leur caractère ethnique et géographique, puisqu'il s'agissait de Juifs nés à Rome. Ces Juifs romains, dont la langue maternelle n'était plus l'araméen ${ }^{32}$, se regroupèrent donc en une communauté afin de se différencier des Juifs qui affluèrent en grand nombre vers la ville de Rome durant la période flavienne ${ }^{33}$. Selon H. Leon, l'oubli de la langue maternelle n'est pas à l'origine de la décision des Juifs de Rome pour fonder une synagogue à part; la langue utilisée par les membres d'une même synagogue ne peut expliquer à elle seule sa fondation, puisque les inscriptions funéraires de Rome montrent que les membres de la synagogue des Hébreux comme ceux de la synagogue des Vernaculi utilisaient indistinctement le grec ${ }^{34}$.

Ces différentes sources épigraphiques ont permis aux chercheurs de reconstituer l'organisation de la synagogue des Vernaculi à Rome, dont voici en quelques lignes les principales fonctions communautaires :

1) «L'archisynagogue » est le chef religieux de la synagogue il préside les assemblées et veille sur l'observance des préceptes de la Loi ${ }^{35}$. P. Van der Horst l'identifie au resh haknesset ראש הכנסת de la littérature rabbinique ${ }^{36}$. D'après l'inscription de Théodote fils de Ouettènos que nous étudierons par la suite plus en détail, «l'archisynagogue » pourrait avoir aussi eu en charge la construction de l'édifice cultuel rattaché à une même

\footnotetext{
${ }^{30}$ P. van der Horst, Ancient Jewish Epitaph, op. cit., p. 88.

${ }^{31}$ Cf. H. J. Leon, The Jews, op. cit., p. 155. Conclusion acceptée par P. van der Host, Ibid.

32 Pour A. Momigliano, cité dans H. J. Leon, Ibid., p. 156, n. 2, les membres de la synagogue des Vernaculi parlaient le latin.

33 E. M. Smallwood., The Jews under Roman Rule, op. cit., p. 522.

${ }^{34}$ H. J. Leon, The Jews, op. cit., p. 155.

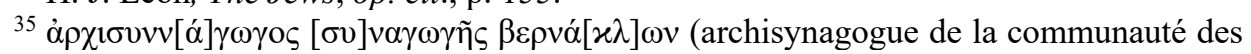
Vernaculi), CIJ, I 383, op.cit., p. 298. Pour une discussion détaillée du rôle des archisynagogoi cf. Tessa Rajak et David Noy, «Archisynagogoi : Office, Tittle, and Status in the Greco-Jewish synagogue », Journal of Roman Studies, n 83, 1993, p. 7593 ; L. Levine, The Ancient Synagogue, op. cit., p. 415-427. Dans les écrits du Nouveau Testament c'est « l'archisynagogue » qui veille sur le respect des préceptes de la Loi et entre en conflit avec Jésus et la doctrine des premiers chrétiens, cf. Lc 13, 14 et Act. Ap. $18,17$.

${ }^{36}$ P. van der Horst, Ancient Jewish Epitaphs, op. cit., p. 92. Cf. TB Pesahim, 49b.
} 
communauté $^{37}$. Cependant ce titre n'est pas spécifique à l'organisation de la synagogue juive et revient souvent dans les associations païennes du monde grec pour désigner le président d'une association.

2) L'archonte est un des membres de la « gerousia » ou conseil des anciens qui dirigeait la communauté ${ }^{38}$. Pour J-B Frey, il n'est pas possible de définir le nombre d'archontes responsables d'une même communauté39. Cette fonction se retrouve sur deux inscriptions épigraphiques de la synagogue des Vernaculi à Rome ${ }^{40}$. Margaret Williams suggère que la fonction d'un ou plusieurs " archonte à vie » indique l'ancienneté de la communauté des Vernaculi dans la ville de Rome ${ }^{41}$.

3) L'une des inscriptions renseigne aussi sur « le père de la synagogue » des Vernaculi, titre honorifique réservé, selon Romano Penna, à des hommes particulièrement méritant occupant une fonction d'administrateur des biens de la synagogue ${ }^{42}$.

4) Enfin la synagogue des Vernaculi disposait également d'un secrétaire faisant fonction de greffier et qu'il ne faut pas confondre avec la fonction du scribe (sofer), telle qu'elle est mentionnée dans les écrits du Nouveau Testament ou de la tradition rabbinique ${ }^{43}$.

Malgré son nom évocateur cette communauté n'était donc pas constituée d'anciens esclaves mais plutôt de Juifs nés à Rome. Cette hypothèse est confirmée par les noms propres apparaissant dans les

${ }^{37}$ CIJ, II 1404.

${ }^{38}$ H. J. Leon, The Jews, op. cit., p. 176 ; P. van der Horst, Ancient Jewish Epitaphs, op. cit., p. 90-91. Également : Mauro Pesce, Paolo e gli Arconti a Corinto, Brescia, Paiedeia Editrice, 1977, p. 277-320 ; Margaret H. Williams, « The Structure of Roman Jewry ReConsidered: Were the Synagogues of Ancient Rome Entirely Homogeneous?», Zeitschrift für Papyrologie und Epigraphik, $\mathrm{n}^{\circ}$ 104, 1994, p. 129-141; L. Levine, The Ancient Synagogue, op. cit, p. 427-428.

${ }^{39} \mathrm{~J}-\mathrm{B}$ Frey, « Le Judaïsme à Rome aux premiers temps de l’Église », Biblica, nº12, 1931, p. 132.

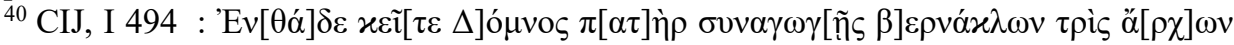
$\kappa(\alpha i) \delta i \varsigma[\varphi] \rho o v \tau[1 \sigma \tau \hat{]}] \varsigma$ (ici repose, Domnos père de la communauté des Vernaculi, trois

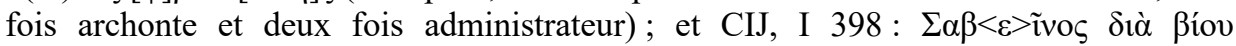
$\beta \varepsilon \rho v \alpha x \lambda \eta \sigma i ́ \omega v$ ( Sabinos, archonte à vie des Vernaculi); selon M. H. Williams la formule " $\delta i \alpha ̀$ Bíov» fait référence aux archontes qui étaient désignés à vie, "The Structure of Roman Jewry », op. cit., p. 131, n. 14.

${ }^{41}$ M. H. Williams, Ibid., p. 141.

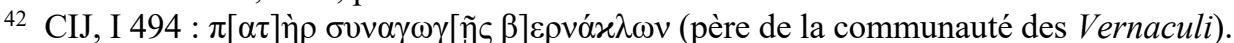
Cf. R. Penna, «Les Juifs à Rome », op. cit., p. 330. J-B Frey compare ce titre à celui des Patronius dans les collegia romains, «Le Judaïsme à Rome », op. cit., p. 133. Sur les fonctions des « pater synagogae », cf. J. Juster, Les Juifs dans l'empire romain, I, op. cit., p. 448-449 ; H. J. Leon, The Jews, op. cit., p. 186-188.

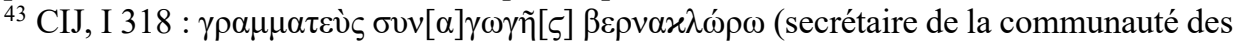
Vernaculi). 
sources épigraphiques et qui ne révèlent aucun lien avec le monde de l'esclavage ou celui des affranchis. Les différentes fonctions indiquées dans les inscriptions relevant de cette communauté permettent de mettre en relief une organisation sociale et une vie religieuse des Juifs de Rome.

Abordons maintenant l'inscription de Théodote fils de Ouettènos, découverte à Jérusalem au cours de l'hiver 1913-1914, faisant allusion à une synagogue définie comme un édifice cultuel et dont le nom du bâtisseur paraît évoquer une origine servile ${ }^{44}$. Cette inscription a fait l'objet de nombreux travaux de recherche déjà anciens qui la datent entre le $\mathrm{I}^{\text {er }}$ siècle av. J-C et la fin du $\mathrm{II}^{\mathrm{e}}$ siècle de notre ère ${ }^{45}$.

Voici le texte de cette inscription :

\begin{abstract}
Théodote fils de Ouettenos, prêtre et archisynagogue, fils d'archisynagogue petit-fils d'archisynagogue, a construit la synagogue, pour la lecture de la Loi et pour l'enseignement des commandements, ainsi que l'hôtellerie et les chambres et les aménagements des eaux, pour servir d'auberge à ceux qui, (venus) de l'étranger,

en auraient besoin, synagogue qu'avaient fondée ses pères et les anciens et les Simonides ${ }^{46}$
\end{abstract}

Que savons-nous donc de l'identité du bâtisseur de la synagogue? Son nom Théodote est bien connu dans l'onomastique gréco-romaine juive, équivalant à Jonathan, Nathan ou Nathaniel ${ }^{47}$. Son patronyme Ouettènos est un nom propre de physionomie latine qui pourrait correspondre à Vettenus ou Vettienus. Ce nom ne figure pas dans les listes des noms juifs de l'époque romaine, recueillis dans les travaux de Tal $\operatorname{Ilan}^{48}$. L'usurpation d'un gentilice romain étant un délit selon la loi, ce nom ne peut donc avoir été porté par un Juif que si celui-ci était un affranchi ou un descendant d'affranchi.

Si Théodote était lui-même affranchi d'un certain Vettenus, il aurait, sans aucun doute, adopté le prcenomen et le nomen de ses patrons auquel il aurait ajouté son nom d'esclave, comme cela était en usage à Rome.

\footnotetext{
${ }^{44}$ Louis-Hugues Vincent, « Découverte de la 'Synagogue des Affranchis' à Jérusalem », Revue Biblique 30, 1921.

${ }^{45}$ Pour un débat détaillé sur la datation de l'inscription, cf., J. S. Kloppenborg, « The Theodotos Synagogue Inscription and the Problem of First-Century Synagogues Buildings », dans J. H. Charlesworth (ed.), Jesus and Archeology, Cambridge, Eerdmans, 2006, p. 253-263.

${ }^{46}$ Lecture et traduction de J-B. Frey, CIJ, II 1404, op. cit., p. 332-334.

${ }^{47}$ Cf. Tal Ilan, Lexicon of Jewish Names in Late Antiquity, I (Palestine, 330 BCE-200 CE), Tübingen, Mohr Siebek, 2002, p. 285-286.

${ }^{48}$ Ibid., p. 325-345.
} 
Mais la forme mentionnée sur l'inscription, Théodote fils de Ouettènos, et plus précisément l'utilisation d'un patronyme signalent que le bâtisseur de la synagogue n'était pas un esclave affranchi mais un Juif, né de condition libre et descendant d'un esclave affranchi de la gens romaine Vettena.

Parmi les ancêtres de Théodote, quel fut le premier à porter le nom de Vettenus?

Une majorité de chercheurs fait remonter la construction de la synagogue de Théodote fils de Ouettènos, à une date à peine antérieure à la destruction de Jérusalem (70 ap. J-C.) Selon Charles ClermontGanneau, suivi par beaucoup d'autres, le premier affranchi aurait ainsi pu être l'un des captifs juifs de Pompée, rendu à la liberté vers 60 av. J-C ${ }^{49}$.

Ce qui suppose que les descendants de cet affranchi, et tout spécialement Théodote, avaient fait suffisamment fortune pour parvenir à financer un tel édifice à Jérusalem comprenant, en plus de la synagogue, l'aménagement d'une auberge.

Selon une autre hypothèse envisagée par John Kloppenborg, Théodote et ses aïeux n'étaient pas d'anciens esclaves mais des Juifs de la diaspora portant des noms grecs et latins ${ }^{50}$. Cette conclusion s'appuie principalement sur une autre inscription d'époque romaine retrouvée à Benghazi, ville de la Cyrénaïque, qui indique que le père d'un des donateurs de la synagogue était un certain Cornelius ${ }^{51}$.

Abordons maintenant la question concernant la fonction d' " archisynagogue » et le statut d'affranchi. Selon l'inscription nommant Théodote, son père et son grand-père (hypothétiquement d'origine servile) du titre honorifique d'«archisynagogue », il semblerait qu'il n'existe aucune incompatibilité entre le statut d'ancien esclave et cette fonction religieuse. Cependant, s'il est clair qu'ayant en charge la construction de la synagogue, Théodote exerçait la fonction d' " archisynagogue », on ne peut pas conclure de façon certaine qu'il en fut de même pour ses ancêtres.

Il apparaît néanmoins, à suivre l'inscription de Théodote fils de Ouettènos, qu'un Juif affranchi fut réhabilité dans ses fonctions religieuses, bénéficiant dès lors d'un accès aux charges synagogales. Cette déduction est confirmée par la littérature rabbinique autour du verset du Lévitique XXV, 27 selon lequel un esclave libéré « rentrera dans son

\footnotetext{
${ }^{49}$ Charles Clermont-Ganneau, « Découverte à Jérusalem d'une synagogue de l'époque hérodienne », Syria 1, 1920.

50 J. S. Kloppenborg, « The Theodotos Synagogue », op. cit., p. 266.

${ }^{51}$ Gert Lüderitz, Corpus Jüdischer Zeugnisse aus der Cyrenika, $\mathrm{n}^{\circ} 72$, Wiesbaden, Ludwig Reichert, 1983, p. 156, 1. 11.
} 
patrimoine », ce qui inclut, d'après l'interprétation de Louis-Hugues Vincent, une restitution de ses droits, tant civiques que familiaux ${ }^{52}$.

Pour résumer ce paragraphe sur les synagogues dont les noms relèvent d'esclaves ou d'affranchis :

1) Malgré un nom révélateur, la synagogue des Vernaculi ne désigne pas une communauté d'esclaves mais une communauté de Juifs nés à Rome. Il semblerait donc qu'aucune communauté de la Rome antique n'ait été fondée sur la base d'un statut servile.

2) Une synagogue dont le fondateur était descendant d'affranchis, comme celle désignée par l'inscription de Théodote fils de Ouettènos, n'était pas réservée à un public ayant des origines exclusivement serviles.

3) Il n'existe aucune incompatibilité entre les fonctions synagogales et la situation d'affranchi.

Ces conclusions indiquent qu'à la fin de la période du second Temple, des Juifs nés de condition libre comme ceux libérés de l'esclavage pouvaient faire partie d'une même communauté et fréquenter le même édifice cultuel.

\section{Les Affranchis et leur synagogue dans Act. Ap. VI, 9}

Dans le Nouveau Testament, Étienne apparaît comme un Juif hellénistique qui a reconnu le message de Jésus. Il débat avec des Juifs hiérosolomytains appartenant à différentes synagogues: "Alors intervinrent des gens de la synagogue dite des Affranchis et des Cyrénéens et des Alexandrins et d'autres de Cilicie et d'Asie ${ }^{53}$. Il

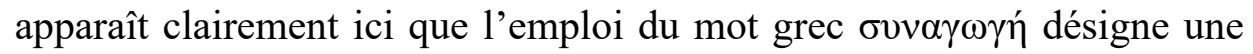
assemblée ou une congrégation et non un édifice ${ }^{54}$.

La synagogue des Affranchis est nommée en grec synagôgè Libertinov, le mot Libertinoi étant une translitération du latin Libertini «affranchis». Cependant les autres synagogues sont toutes dénommées d'après une origine géographique. Cette observation est à l'origine d'une hypothèse selon laquelle le terme Libertini, employé dans le texte, ne désignerait pas des affranchis $(\Lambda 1 \beta \varepsilon \rho \tau i v \omega v)$ mais plutôt une communauté de Juifs venus de Lybie $\left(\Lambda \_\beta v \sigma \tau i ́ v \omega v\right)$, origine proche des deux autres

\footnotetext{
${ }^{52}$ L-H. Vincent, « Découverte de la 'Synagogue des Affranchis'», op. cit., p. 271. TB, Makkot 13a.

${ }_{53}$ Act. Ap. VI, 9. Ma traduction.

${ }^{54}$ Bruno W. W. Dombrowski, «Synagōge in Acts $6: 9 »$, Zdzislav J. Kapera (ed.), Intertestamental Essays in Honour of Józef Tadeusz Milik, Kraków, The Enigma Press, 1992, p. 57-58.
} 
noms géographiques indiqués, Alexandrie et Cyrène ${ }^{55}$. Néanmoins nous préférons souscrire à l'opinion commune et identifier le terme Libertini à d'anciens esclaves.

Indiquons aussi que de nombreuses discussions ont été menées par les chercheurs pour savoir s'il était question d'une seule ou bien de plusieurs synagogues ${ }^{56}$. Ainsi, Joachim Jeremias qui s'appuie sur la littérature rabbinique, suggère que les diverses synagogues mentionnées ne sont, en fait, qu'une seule congrégation de Juifs hellénistiques appartenant à la «synagogue des Alexandrins", ou à celle «des Tarsiens $\gg{ }^{57}$. Selon William Denton, il s'agissait de deux synagogues, l'une « des Affranchis » constituée de Juifs de Cyrène et d'Alexandrie et l'autre constituée de Juifs d'Asie Mineure ${ }^{58}$. Dans cet article on retiendra l'hypothèse d'Emil Schürer, selon laquelle le verset Act. Ap. VI 9 fait état de cinq synagogues distinctes, insistant sur leurs origines géographiques pour quatre d'entre elles, tandis que pour la cinquième le texte évoque un statut social relevant d'une origine servile ${ }^{59}$. Il semble aussi que les membres de ces synagogues, originaires de la diaspora, parlaient vraisemblablement le grec $^{60}$.

La présence à Jérusalem des différentes communautés signalées dans Act. Ap. VI 9 est attestée par d'autres sources. Nous savons ainsi que les Juifs de Cyrène et ceux d'Égypte avaient l'habitude de se rendre à Jérusalem $^{61}$. Les écrits de Philon d'Alexandrie renseignent aussi sur le pèlerinage des Juifs d'Alexandrie au Temple de Jérusalem ${ }^{62}$. À propos des Juifs de Cilicie, citons l'exemple de Paul qui vint de Tarse à Jérusalem,

\footnotetext{
${ }^{55}$ Charles K. Barrett, A Critical and Exegetical Commentary on the Acts of the Apostles, I, Edinburg, T\&T Clark LTD, 1994, p. 323.

${ }^{56}$ Ibid., p. 324.

${ }^{57}$ Joachim Jeremias, Jerusalem in the Times of Jesus, Philadelphia, Fortress Press, 1975, p. 65-66. Les sources rabbiniques sur « la synagogue des Alexandrins » : Tosefta Méguila II 56-57; TJ, Méguila III 1, 73d, 1. 35. Dans le Talmud Babylonien, à la place de « synagogue des Alexandrins », on trouve « synagogue des Tarsiens », TB, Méguila 26 a.

58 William Denton, Commentary on the Acts of the Apostles, I, London, Bell, 1874, p. 187-188.

${ }^{59}$ Emil Schürer, The History of the Jewish People in the Age of Jesus Christ [175 B.C135 A.D], revised and edited by G. Vermes, F. Millar \& M. Black, II, Edinburgh, Clark, 1987 ( 3 édition), p. 428 et note 8.

${ }^{60}$ Les Juifs de Cyrène sont de nouveau mentionnés dans Act. Ap. XI 20 parmi ceux qui, venus à Antioche, communiquent avec des gens de langue grecque.

${ }^{61}$ Act. Ap. II 10 ; Mc XV 21 ; Lc XXIII 26.

62 Philon d'Alexandrie, Fragments De Providentia, dans Eusebe de Césarée, La préparation évangelique, VIII 14, 64, traduit par Guy Shroeder \& Édouard Des Places, Paris, Sources Chrétiennes, p. 174-177.
} 
pour y suivre des études dans l'école de Gamaliel l'Ancien ${ }^{63}$. Quant aux Juifs d'Asie mineure, leur présence est attestée dans le Temple lors de la fête de la Pentecôte alors qu'ils haranguent les foules pour se saisir de Paul $^{64}$.

Ce contexte permet donc d'accepter les conclusions de Joachim Jeremias au sujet des synagogues indiquant une origine géographique : les Juifs de différentes diasporas étaient désireux de posséder dans la ville sainte leur centre particulier où ils pouvaient, en dehors du service liturgique dans le Temple, s'adonner à d'autres exercices religieux et à l'étude de la Loi ${ }^{65}$.

Si la synagogue des Affranchis réunissait d'anciens captifs, asservis à Rome puis libérés par leurs maîtres, il se pourrait donc que cette communauté ait été réservée aux Juifs de la diaspora romaine. Pour quelle raison alors les membres de cette communauté voulaient-ils préserver le souvenir de leur ancien esclavage à travers le nom de leur synagogue ?

On pourrait envisager une autre hypothèse et identifier ces affranchis non à d'anciens captifs juifs mais plutôt à d'anciens esclaves païens ayant embrassé la religion de leurs maîtres juifs. Citons, à ce propos, le témoignage de Tacite qui précise qu'à l'époque de l'empereur Tibère (1437 ap. J-C), des hommes de la classe des Libertini avaient adopté à Rome les «superstitions judaïques", évoquant par là un certain lien entre affranchis et prosélytes ${ }^{66}$.

Ces mêmes prosélytes romains avaient d'ailleurs l'habitude de se rendre en pèlerinage dans la ville de Jérusalem, comme le signale le passage de Act. Ap. II 10. On peut aussi rappeler les actes d'affranchissement du Bosphore, évoqués au début de cet article, qui confirment la fréquentation assidue d'affranchis païens dans les synagogues de leurs anciens maîtres. Cependant maîtres juifs et anciens esclaves païens fréquentaient alors la même synagogue.

Les données dont nous disposons ne nous permettent donc pas de définir avec exactitude l'identité des membres de la synagogue des Affranchis. Nous admettrons cependant que la proximité de la synagogue des Affranchis avec d'autres synagogues fréquentées par des Juifs de la diaspora (Cyrène, Alexandrie, Cilicie et Asie Mineure) est susceptible de révéler l'origine étrangère (diasporique) des fidèles de cette synagogue.

\footnotetext{
${ }^{63}$ Act. Ap. XXII, 3.

${ }^{64}$ Act. Ap. XXI, 27.

${ }^{65}$ J. Jeremias, Jerusalem, op. cit., p. 62.

${ }^{66}$ Tacite, Annales II 85, 4.
} 
Nous conclurons notre étude sur les affranchis et leur synagogue à la fin de la période du second Temple en proposant ce qui suit :

1) Les synagogues tenaient une place dans la vie juive et cela même avant

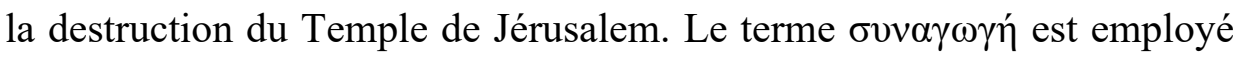

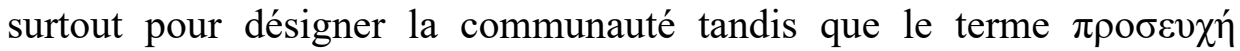
s'identifie au lieu de prière dès une époque très reculée.

2) La synagogue est le centre de l'étude de la Loi juive, on y rend aussi la justice et on y exécute les sentences. L'affranchissement d'esclaves païens dans la synagogue indique le caractère sacré de cette institution aux yeux de Juifs de la diaspora.

3) Bien que la synagogue des Vernaculi indique par son nom une origine servile, ses membres étaient en fait des Juifs nés à Rome désireux de se distinguer d'autres Juifs qui affluaient vers la ville. Les deux synagogues attestant de la présence d'anciens esclaves, celle désignée par l'inscription de Théodote fils de Ouettènos et l'autre mentionnée dans Act. Ap. VI 9, se trouvent dans la ville de Jérusalem et font toutes deux références à une institution pour Juifs de la diaspora.

4) Nous n'avons pas pu définir avec exactitude le parcours et l'identité de ces Juifs qui se réunissaient dans la synagogue des Affranchis, d'après l'auteur des Actes des Apôtres. S'agissait-il de Juifs faits captifs à Rome et libérés de retour à Jérusalem ou bien d'esclaves païens qui avaient embrassé la religion de leurs maîtres juifs ? Dans l'état actuel des recherches historiques, cette question reste sans réponse. 